\title{
A SUITABLE GEOMETRICAL MODEL FOR THE VERIFICATION OF KNOOP INDENTERS WITH GAL-INDENT OPTICAL SYSTEM
}

\author{
A. Prato ${ }^{1}$, C. Origlia $^{1}$, A. Germak ${ }^{1}$ \\ ${ }^{1}$ INRiM - Istituto Nazionale di Ricerca Metrologica, Torino, Italy, a.prato@inrim.it
}

\begin{abstract}
:
ISO 4545-2 and 4545-3 of Knoop hardness tests require the geometrical verification of the indenter. INRiM hardness laboratory developed a specific measuring system, commercialized by GalileoLTF $尺$ as Gal-Indent optical system, which is used for the verification of Vickers indenters. This system is able to measure the vertex angles of the indenter between two opposite faces, and the four quadrilateral base angles. Using such quantities as inputs of a suitable geometrical model, the geometry of Knoop indenters can be verified. This work deals with the description of the system and the geometrical model.
\end{abstract}

Keywords: Knoop, hardness, optical system, indenter, Gal-indent.

\section{INTRODUCTION}

Knoop indenter is a rhombic-based pyramidal diamond that produces an elongated diamondshaped indent. The angles from the opposite edges at the vertex of the diamond pyramid of the indenter are $172.5^{\circ}$ and $130^{\circ}$, and the ratio between long and short diagonals is approximately 7.11 to 1 . This entails that the angles of the rhombic base are $164^{\circ}$ and $16^{\circ}$, and the angles between the two opposite faces of the vertex are $129.57^{\circ}$. ISO $4545-2$ and 4545-3 [1,2] specify the requirements of the indenters with different tolerances. The second is more restrictive since it refers to the calibration of reference blocks. The tolerance for the angle of $172.5^{\circ}$ is $\pm 0.1^{\circ}$ in both documents, whereas for $130^{\circ}$ the tolerances are $\pm 1^{\circ}$ and $\pm 0.1^{\circ}$, respectively. Furthermore, the angle between the axis of the diamond pyramid and the axis of the indenter holder (normal to the seating surface), namely tilt angle, shall not exceed $0.5^{\circ}$ and $0.3^{\circ}$, respectively. The device used for the verification shall have a maximum expanded uncertainty of $0.07^{\circ}$. INRiM hardness laboratory developed a specific measuring system, commercialized by the Galileo-LTF® as Gal-Indent optical system for the verification of the geometrical characteristics of Vickers indenters [35]. This system is able to directly measure the main geometrical parameters of a Vickers indenter required by the standard, i.e. the two vertex angles (nominally $136^{\circ}$ ) between two opposite faces and the four angles of the square base, both with an expanded uncertainty of $0.05^{\circ}$. Using these measured quantities as input of a suitable geometrical model, the possibility to verify Knoop indenters is investigated. This paper deals with the description of the system and the geometrical model.

\section{THE GAL-INDENT OPTICAL SYSTEM}

In INRiM hardness laboratory a specific measuring system, commercialized by GalileoLTF $®$ as Gal-Indent optical system (Figure 1), was developed and is currently used for the verification of Vickers indenters. The system is also adopted by different National Metrological Institutes (NMI) and calibration laboratories around the world. The Galileo-LTF® Gal-Indent optical system is based on Mirau interferometry. The geometrical characteristics assure that all mechanical parts are perfectly aligned and that the indenter-holder axis is perpendicular to the lens of the system. The Knoop indenter is simultaneously rotated around the axis passing through the indenter vertex normal to the plane containing the indenter-holder axis and the optical lens axis, and around the indenter-holder axis, until a lateral face is parallel to the plane of the microscope lens by observing the interference fringes. These two rotations are measured by means of two angular encoders [6]. Rotations around the indenter-holder axis represent the measurement of the angles between two consecutive faces, i.e. the quadrilateral base angles $\varphi$ (two angles nominally $164^{\circ}$ ) and $\tau$ (two angles nominally $16^{\circ}$ ); whereas, rotations around the axis normal to the plane containing the indenter-holder axis and the optical lens axis represent the measurement of the supplementary angles for each lateral face $\omega$ (nominally $25.22^{\circ}$ ) from which the angles between two opposite faces $\theta$ (nominally $129.57^{\circ}$ ) are easily obtained [7]. Such values, used as input in a suitable geometrical model, allow to evaluate the angles from the opposite edges at the vertex $\alpha$ and $\beta$, 
nominally equal to $172.5^{\circ}$ and $130^{\circ}$, respectively, and the tilt angle $\delta$, nominally equal to $0^{\circ}$.
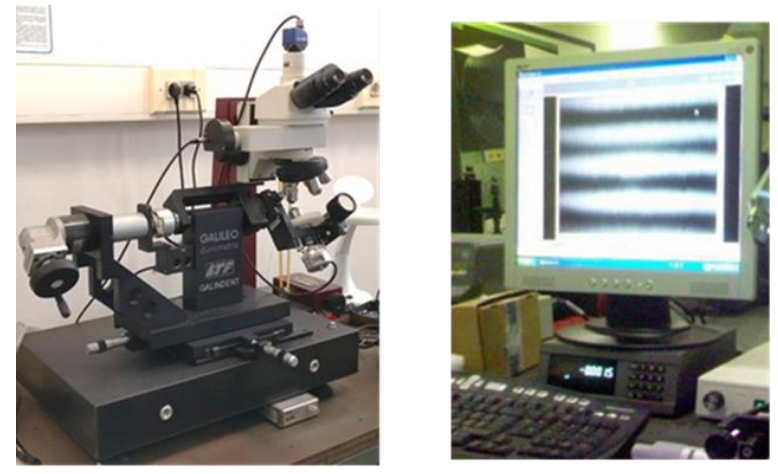

Figure 1: The Galileo-LTF® Gal-Indent optical system.

\section{THE GEOMETRICAL MODEL}

\subsection{Evaluation of the tilt angle}

The geometry of a real Knoop indenter with four generic faces (A, B, C, D) is schematically depicted in Figure 2. $x y z$ and $x^{\prime} y^{\prime} z^{\prime}$ coordinate systems correspond, respectively, to the diagonals of the Knoop indenter rhombic base, and to the optical reference system that is perpendicular to the perimeter of two opposite faces. Therefore, the angle between $x$ - and $y$-axis is nominally $90^{\circ}$, whereas the angle $\sigma_{A B}$ between $x^{\prime}$ - and $y^{\prime}$-axis is nominally $164^{\circ}$. Since the tilt angle $\delta$ is not exactly $0^{\circ}$, an angle $\gamma$ between the projection of the pyramid vertex on $z=0$ plane and $x^{\prime}$-axis appears.

For each $j$-th face $(j=\mathrm{A}, \mathrm{B}, \mathrm{C}, \mathrm{D})$, the intersection between an optical reference axis ( $x^{\prime}$ - or $y^{\prime}$-axis) and the base perimeter is identified by point $\mathrm{H}_{\mathrm{j}}$, whereas the intersection with $x$ - and $y$-axis are identified by points $S_{j}$ and $P_{j}$, respectively (thus $S_{A} \equiv S_{D}, S_{B} \equiv S_{C}$, $\left.\mathrm{P}_{\mathrm{A}} \equiv \mathrm{P}_{\mathrm{B}}, \mathrm{P}_{\mathrm{C}} \equiv \mathrm{P}_{\mathrm{D}}\right)$. The pyramid vertex $\mathrm{V}$ is arbitrally placed on $z=1$. A cross-section of a real Knoop indenter along $x^{\prime} z^{\prime}$ optical system plane is also shown in Figure 3. The quadrilateral base angles $\varphi_{i}$ and $\tau_{i}(i=1,2)$, nominally $164^{\circ}$ and $16^{\circ}$, respectively, and the supplementary angles of each $j$-th lateral face (A, B, C, D) along $x^{\prime}$ and $y^{\prime}$-axis, $\omega_{j}$, nominally $\left(180^{\circ}-129.57^{\circ}\right) / 2 \approx 25.22^{\circ}$, are measured by means of the optical system previously described.
From quadrilateral base angles measurements $\varphi_{i}$ and $\tau_{i}(i=1,2)$, in order to take into account possible asymmetries of the rhombic-base, the mean angle $\rho_{j}=\widehat{\mathrm{H}}_{\mathrm{J}} \mathrm{OS}_{\mathrm{j}}$ between $x y$ and $x^{\prime} y^{\prime}$ reference systems, for each $j$-th indenter face, can be evaluated according to

$\rho_{j}=\frac{\left(90-\frac{\varphi_{j}}{2}\right)+\frac{\tau_{j}}{2}}{2}$

where $\varphi_{\mathrm{A}}=\varphi_{\mathrm{D}}=\varphi_{1}, \varphi_{\mathrm{B}}=\varphi_{\mathrm{C}}=\varphi_{2}, \tau_{\mathrm{A}}=\tau_{\mathrm{B}}=\tau_{1}, \tau_{\mathrm{C}}=\tau_{\mathrm{D}}=$ $\tau_{2}$.

In this way, considering the mean value $\rho=$ $\sum_{j=1}^{4} \rho_{j} / 4$ among the four faces, the angle $\sigma_{A B}$ between $x^{\prime}$ - and $y^{\prime}$-axis can be obtained according to equation (2).

$\sigma_{A B}=180-2 \rho$

From the measurement of the supplementary angles $\omega_{j}$ of each $j$-th lateral face, the two vertex angles $\theta_{\mathrm{x}^{\prime}}$ and $\theta_{\mathrm{y}^{\prime}}$ and the pyramid tilt angles $\delta_{\mathrm{x}^{\prime}}$ and $\delta$ y', along $x^{\prime}$ - and $y^{\prime}$ - axis, can be calculated according to equations (3) and (4), respectively.

$$
\begin{aligned}
& \theta_{x^{\prime}}=180-\left(\omega_{\mathrm{A}}+\omega_{\mathrm{C}}\right) \\
& \theta_{y^{\prime}}=180-\left(\omega_{\mathrm{B}}+\omega_{\mathrm{D}}\right) \\
& \delta_{x^{\prime}}=\frac{\omega_{\mathrm{A}}-\omega_{\mathrm{C}}}{2} \\
& \delta_{y^{\prime}}=\frac{\omega_{\mathrm{B}}-\omega_{\mathrm{D}}}{2}
\end{aligned}
$$

By decomposing the pyramid tilted axis vector $\mathbf{v}$ along non-orthogonal $x^{\prime} z^{\prime}$ and $y^{\prime} z^{\prime}$ planes, according to Figure 4, equation (5) is derived. Successively, implementing the equations of non-orthogonal systems (Figure 5) and using equation (5), equations (6)-(8) and equations (9)-(10) can be derived.

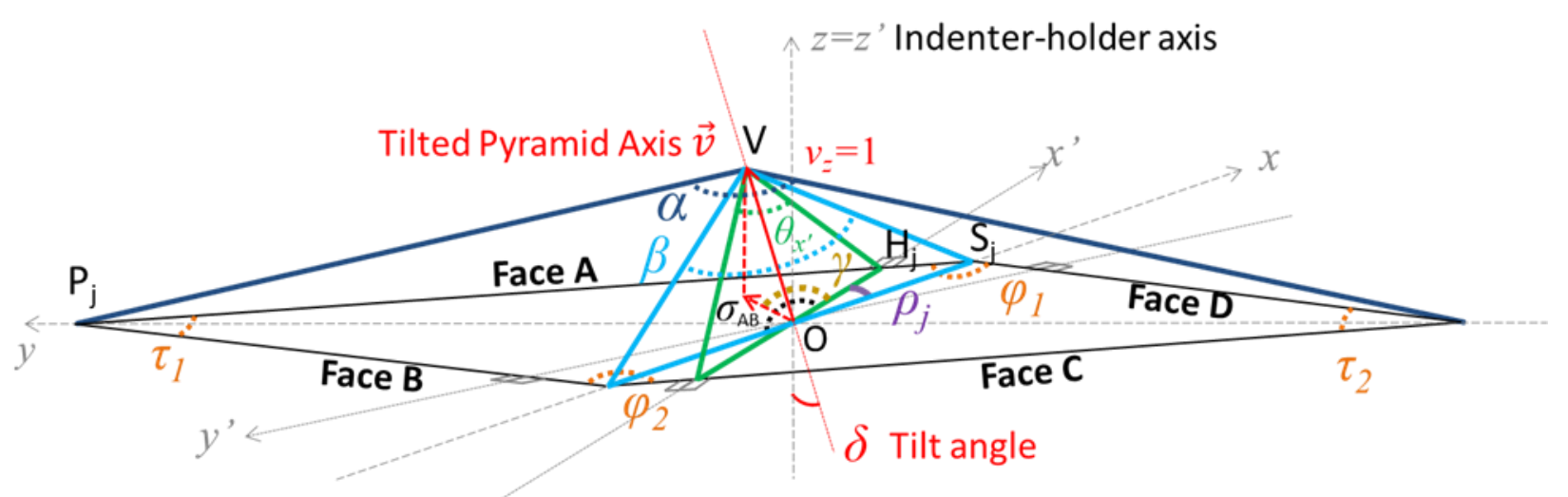

Figure 2: 3-D schematic representation of a real Knoop indenter rhombic-based pyramid 


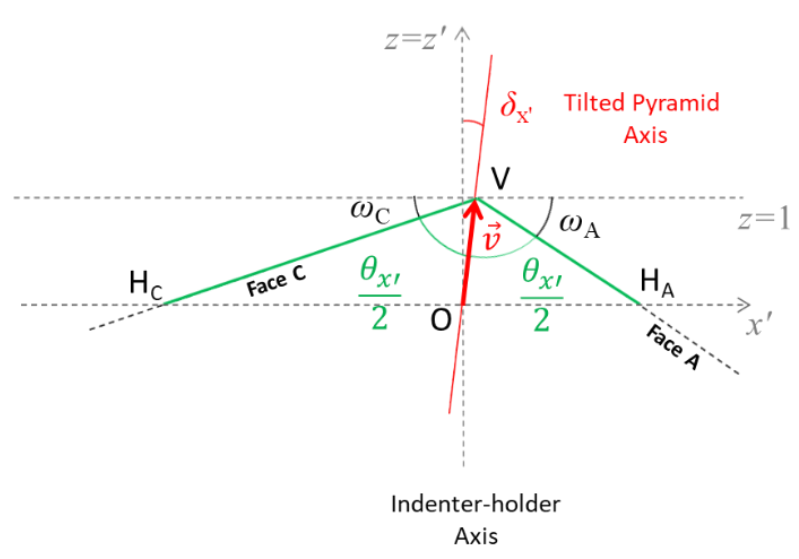

Figure 3: Cross-section of a real Knoop indenter along $x^{\prime} z^{\prime}$ optical reference system plane.

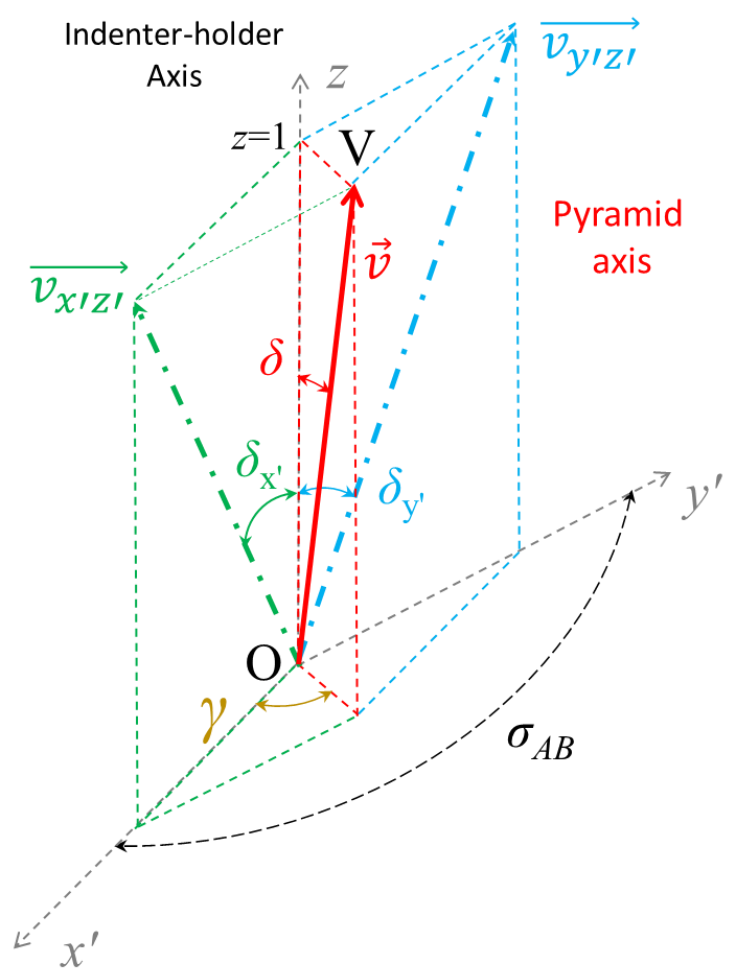

Figure 4: Decomposition of the tilted pyramid axis vector $\mathbf{v}$ and the associated angles along $x^{\prime}$ - and $y^{\prime}$ - axis.

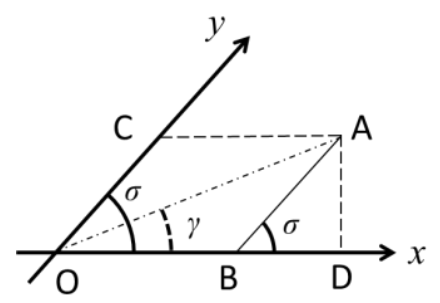

$\left\{\begin{array}{l}\overline{O A} \cos \gamma=\overline{O B}+\overline{O C} \cos \sigma \\ \overline{O A} \sin \gamma=\overline{O C} \sin \sigma\end{array}\right.$

Figure 5: Generic non-orthogonal reference system with relevant equations.

$\|\mathbf{v}\| \cos \delta=\left\|\mathbf{v}_{\boldsymbol{x}^{\prime} \mathbf{z}^{\prime}}\right\| \cos \delta_{\mathrm{x}^{\prime}}=$

$$
\begin{aligned}
& =\left\|\mathbf{v}_{y^{\prime} \mathbf{z}^{\prime}}\right\| \cos \delta_{\mathbf{y}^{\prime}} \\
& \|\mathbf{v}\| \sin \delta \cos \gamma=\|\mathbf{v}\| \frac{\cos \delta}{\cos \delta_{x^{\prime}}} \sin \delta_{x^{\prime}}+ \\
& +\|\mathbf{v}\| \frac{\cos \delta}{\cos \delta_{y^{\prime}}} \sin \delta_{y^{\prime}} \cos \sigma_{A B} \\
& \sin \delta \cos \gamma=\frac{\cos \delta}{\cos \delta_{x^{\prime}}} \sin \delta_{x^{\prime}}+ \\
& +\frac{\cos \delta}{\cos \delta_{y^{\prime}}} \sin \delta_{y^{\prime}} \cos \sigma_{A B} \\
& \tan \delta \cos \gamma=\tan \delta_{x^{\prime}}+\tan \delta_{y^{\prime}} \cos \sigma_{A B} \\
& \|\mathbf{v}\| \sin \delta \sin \gamma=\left\|\mathbf{v}_{\mathbf{y}^{\prime} \mathbf{z}^{\prime}}\right\| \sin \delta_{y^{\prime}} \sin \sigma_{A B} \\
& =\|\mathbf{v}\| \frac{\cos \delta}{\cos \delta_{y^{\prime}}} \sin \delta_{y^{\prime}} \sin \sigma_{A B} \\
& \tan \delta \sin \gamma=\tan \delta_{y^{\prime}} \sin \sigma_{A B}
\end{aligned}
$$

By performing the squared sum of equations (8) and (10), the total tilt angle $\delta$ can be obtained (see equations (11)-(13)):

$$
\begin{aligned}
& \tan ^{2} \delta \cos ^{2} \gamma+\tan ^{2} \delta \sin ^{2} \gamma= \\
& =\tan ^{2} \delta_{x^{\prime}}+\tan ^{2} \delta_{y^{\prime}} \cos ^{2} \sigma_{A B^{+}}+ \\
& +2 \tan \delta_{x^{\prime}} \tan \delta_{y^{\prime}} \cos \sigma_{A B^{+}} \\
& +\tan ^{2} \delta_{y^{\prime}} \sin ^{2} \sigma_{A B} \\
& \tan ^{2} \delta=\tan ^{2} \delta_{x^{\prime}}+\tan ^{2} \delta_{y^{\prime}}= \\
& =+2 \tan \delta_{x^{\prime}} \tan \delta_{y^{\prime}} \cos \sigma_{A B} \\
& \delta=\arctan \sqrt{\tan ^{2} \delta_{x^{\prime}}+\tan ^{2} \delta_{y^{\prime}}}+2 \tan \delta_{x^{\prime}} \tan \delta_{y^{\prime}} \cos \left(\sigma_{A B}\right)
\end{aligned}
$$

By performing the ratio between equations (10) and (8), the angle $\gamma$ can be derived (see equations (14)-(15)):

$$
\begin{aligned}
& \tan \gamma=\frac{\tan \delta_{y^{\prime}} \sin \sigma_{A B}}{\tan \delta_{x^{\prime}}+\tan \delta_{y^{\prime}} \cos \sigma_{A B}} \\
& \gamma=\arctan \left(\frac{\tan \delta_{y^{\prime}} \sin \sigma_{A B}}{\tan \delta_{x^{\prime}}+\tan \delta_{y^{\prime}} \cos \sigma_{A B}}\right)
\end{aligned}
$$

\subsection{Evaluation of the angles between the opposite edges at the vertex}

From the scheme of Figure 2, reminding that the pyramid vertex $\mathrm{V}$ is placed on $z=1$, the vector of the tilted pyramid axis $\mathbf{v}$ referred to the $x y z$ reference system can be written according to equation (16), where $\rho$ is the mean angle between $x^{\prime}$ - and $x$-axis (see equation (2)). 
$\mathbf{v}=[\tan (\delta) \cos (\gamma+\rho)$,

$\tan (\delta) \sin (\gamma+\rho), 1]$

Considering the triangle $\mathrm{OH}_{\mathrm{j}} \mathrm{V}$ in Figure 2 and Figure 3 for each $j$-th indenter face (A, B, C, D), given that $\widehat{\mathrm{OH}_{\mathrm{j}} \mathrm{V}}=\omega_{j}$, and implementing the law of sines, equation (17) is obtained:

$$
\overline{\mathrm{OH}}_{j}=\|\mathbf{v}\| \frac{\sin \frac{\theta_{j}}{2}}{\sin \omega_{j}}
$$

where $\theta_{\mathrm{A}}=\theta_{\mathrm{C}}=\theta_{\mathrm{x}^{\prime}}$ and $\theta_{\mathrm{B}}=\theta_{\mathrm{D}}=\theta_{\mathrm{y}^{\prime}}$.

In this way, equations (18) and (19) are derived. The sign of the vector components for the four faces follows the position on the $x y z$ reference system as in Figure 2.

$$
\begin{aligned}
& \mathbf{O S}_{j}=\left(\frac{\overline{\mathrm{OH}}_{j}}{\cos \rho_{j}}, 0,0\right) \\
& \mathbf{O P}_{j}=\left(0, \frac{\overline{\mathrm{OH}}_{j}}{\sin \rho_{j}}, 0\right)
\end{aligned}
$$

Considering the triangle $\mathrm{OVS}_{\mathrm{j}}$, it is obtained that

$$
\widehat{V O S}_{J}=\cos ^{-1} \frac{\mathbf{O S}_{j} \cdot \mathbf{v}}{\|\mathbf{v}\|\left\|\mathbf{O S} \mathbf{S}_{j}\right\|}
$$

Again, by applying the law of sines to triangle $\mathrm{OVS}_{\mathrm{j}}$, it is obtained that

$$
\frac{\|\mathbf{v}\|}{\sin \widehat{O S_{J} V}}=\frac{\left\|\mathbf{O S}_{j}\right\|}{\sin \widehat{O V S_{J}}}=\frac{\left\|\mathbf{v s}_{j}\right\|}{\sin \widehat{V O S}_{J}}
$$

Given that $\widehat{O S_{J} V}=180-\widehat{O V S_{J}}-\widehat{V O S_{J}}$ and with some trigonometric calculations, equation (22) is obtained.

$$
\begin{aligned}
& \sin \widehat{O S_{J} V}=\sin \left(180-\widehat{O V S}_{J}-\widehat{V O S}_{J}\right)= \\
& =\sin {\widehat{O V S_{J}}}_{\operatorname{los}} \cos \overline{V O S}_{J}+\sin \overline{V O S}_{J} \cos \widehat{O V S}_{J}
\end{aligned}
$$

In this way, combining equations (21) and (22), equations (23)-(25) are obtained:

$$
\begin{aligned}
& \frac{\|\mathbf{v}\|}{\left\|\mathbf{O S}_{j}\right\|}=\frac{\sin \widehat{O S_{J} V}}{\sin \widehat{O V S_{J}}} \\
& \frac{\|\mathbf{v}\|}{\left\|\mathbf{O S}_{j}\right\|}= \\
& =\frac{\sin \widehat{O V S}_{J} \cos \widehat{V O S}_{J}+\sin \widehat{V O S}_{J} \cos \widehat{O V S}_{J}}{\sin \widehat{O V S}_{J}}
\end{aligned}
$$

$$
\frac{\|\mathbf{v}\|}{\left\|\mathbf{O S}_{j}\right\|}=\cos \widehat{V O S}_{J}+\frac{\sin \widehat{V O S_{J}}}{\tan \widehat{O V S_{J}}}
$$

and from equation (25), equation (26) is also obtained:

$$
\widehat{O V S}_{J}=\tan ^{-1} \frac{\left\|\mathbf{O S}_{j}\right\| \sin \widehat{V O S}_{J}}{\|\mathbf{v}\|-\left\|\mathbf{O S}_{\boldsymbol{j}}\right\| \cos \overline{V O S}_{J}}
$$

By applying the same calculations from equation (20) onward to triangle $\mathrm{VOP}_{j}$, it is found that,

$$
\widehat{O V P_{j}}=\tan ^{-1} \frac{\left\|\mathbf{O P}_{\mathbf{j}}\right\| \sin \widehat{V O P}_{\jmath}}{\|\mathbf{v}\|-\left\|\mathbf{O P}_{\boldsymbol{j}}\right\| \cos \widehat{V O P}_{\jmath}}
$$

Therefore, considering a single $j$-th indenter face, the angles between two opposite edges can be found according to:

$$
\begin{aligned}
& \alpha_{j}=2 \widehat{O V P_{J}} \\
& \beta_{j}=2 \widehat{O V S_{J}}
\end{aligned}
$$

Averaging the results obtained for each $j$-th indenter face, the angles between two opposite edges, nominally 172.5, equation (30), and $130^{\circ}$, equation (31), are finally obtained:

$$
\begin{aligned}
& \alpha=\frac{\sum_{i=1}^{4} \alpha_{j}}{4} \\
& \beta=\frac{\sum_{i=1}^{4} \beta_{j}}{4}
\end{aligned}
$$

\section{SUMMARY}

ISO 4545-2 and 4545-3 of Knoop hardness tests require the geometrical verification of the indenter. INRiM hardness laboratory developed a specific measuring system, commercialized by GalileoLTF ${ }^{\circledR}$ as Gal-Indent optical system, which is used for the verification of Vickers indenters. This system is also adopted by other NMIs and calibration laboratories around the world. It is able to measure the two vertex angles of the indenter between two opposite faces, and the four angles between two consecutive faces by means of two angular encoders mounted on the optical measuring system. Using such quantities as inputs of the proposed geometrical model described in Section 3, the geometry of Knoop indenters, in particular the angles from the opposite edges at the vertex of Knoop indenters, nominally $172.5^{\circ}$ and $130^{\circ}$, and the angle between the axis of the diamond pyramid and the axis of the indenter holder, nominally $0^{\circ}$, can be verified. 


\section{REFERENCES}

[1] ISO 4545:2017-2 Metallic materials - Knoop hardness test - Verification and calibration of testing machines.

[2] ISO 4545:2017-3 Metallic materials - Knoop hardness test - Calibration of reference blocks

[3] G. Barbato, G. Gori, "Metrological references in Hardness Measurement: a necessary background for industrial Quality Assurance", Proc. of the 5th Congreso Nacional de Metrologia Industrial, Zaragoza, Spain, pp. 139-149, 1991.

[4] G. Barbato, S. Desogus, "Measurement of the spherical tip of Rockwell indenters," Journal of Testing and Evaluation, vol. 16(4), pp. 369-374, 1988.
[5] A. Liguori, A. Germak, G. Gori, E. Messina, "Galindent: the reference metrological system for the verification of the geometrical characteristics of Rockwell and Vickers diamond indenters", Proc. of IMEKO TC3/TC5/TC20, pp. 365-371, 2002.

[6] A. Germak, A. Liguori, C. Origlia, "Experience in the metrological characterization of primary hardness standard machines", Proc. of HARDMEKO 2007, Tsukuba, Japan, pp. 81-89, 2007.

[7] A. Prato, D. Galliani, C. Origlia, A. Germak, "A correction method for Vickers indenters squareness measurement due to the tilt of the pyramid axis", Measurement, vol. 140, pp. 565-571, 2019. 